\title{
Role of polygenic risk in susceptibility to accelerated pubertal onset following chronic stress exposure
}

\author{
Ying Sun ${ }^{1,2}$, Jiao Fang 1,2, Yuhui Wan 1,2, Puyu Su, ${ }^{1,2}$ and Fangbiao Tao ${ }^{1,2}$ \\ ${ }^{1}$ Department of Maternal, Child \& Adolescent Health, School of Public Health, Anhui Medical University and ${ }^{2}$ Anhui \\ Provincial Key Laboratory of Population Health \& Aristogenics, Hefei, Anhui Province, China
}

Correspondence should be addressed to $Y$ Sun Email

yingsun@ahmu.edu.cn

\begin{abstract}
Objective: Previous finding suggests that children growing up under chronic stress tend to experience earlier sexual maturity. The present study aims to examine polygenic risk by experience interaction in predicting pubertal timing, as well as provide insight regarding the relevance of two $\mathrm{G} \times \mathrm{E}$ paradigms.

Design and methods: Data were analyzed from a 3-year prospective puberty cohort in Anhui Province, China. Breast Tanner stage and testicular volume (TV) of 997 children were annually assessed. The polygenic risk score (PRS) was computed based on 17 SNPs for early pubertal timing. Hair cortisol concentrations (HCC) were assessed in the first $3 \mathrm{~cm}$ hair segment as a biological marker of chronic stress.

Results: Comparing with participants under moderate levels of stress as measured by HCC, the puberty-accelerating effects of chronic stress were only observed among girls with moderate $(1.7$ months earlier, $P=0.007)$ and low genetic susceptibility $(2.2$ months earlier, $P<0.001)$ and among boys with high genetic susceptibility $(2.0$ months earlier, $P=0.005$ ). Polygenic differences (PRSs) in age at thelarche was most prominent in those with low levels of stress by $\operatorname{HCC}(9.06,9.36$ and 9.53 years for high, moderate and low PRS, respectively; $F=105.06, P<0.0001)$, while polygenic differences in age at TV $\geq 4 \mathrm{~mL}$ was strongest in those under chronic stress $(10.91,11.06$ and 11.17 years for high, moderate and low PRS, respectively; $F=100.48, P<0.0001$ ).

Conclusion: Chronic stress predicts earlier age at pubertal onset in a sex-specific and genetic background-dependent manner. The bioecological $\mathrm{G} \times \mathrm{E}$ model for girls and diathesis stress model for boys in pubertal timing warrants further investigation.
\end{abstract}

\section{Introduction}

In the past 70 or so years, in many parts of the world, children are entering puberty earlier than ever before. This is associated with a range of adverse health outcomes later in life $(1,2)$. Pubertal timing varies largely between individuals reflecting principally a genetic influence but also nutritional, environmental and socioeconomic factors $(3,4,5,6)$. Accumulating evidence, including one study of ours, suggests that children growing up under high psychosocial stress tend to experience earlier sexual maturity $(7,8,9)$. These associations have been usefully (c) 2019 European Society of Endocrinology Printed in Great Britain interpreted within life-history theory frameworks (10). By contrast to those findings, some studies obtained opposite direction of effects. One study by using the 1958 British birth cohort ( $n=17638$ ) indicated that cumulative neglect in early childhood was associated with late maturation in both sexes and across several pubertal markers (11). Sexual abuse correlated with both late and early menarche, and witnessing abuse was reported to associate with early genitalia development in males. Genome-wide association studies (GWAS)-identified genetic variations underpinning

Published by Bioscientifica Ltd. 
the individual differences in pubertal timing will enable a better understanding of the heterogeneity in association between chronic stress with pubertal timing $(12,13,14)$.

Much of past research is limited by a reliance on retrospective recall on life events or adverse experiences, often via self-report. An important development in the last few years has been the employment of hair cortisol analysis with its unique possibility to assess the longterm systematic levels of cortisol retrospectively (15). Hair cortisol concentration (HCC) is increasingly used as a biological marker of chronic stress and is regarded a promising and nonintrusive method of detecting individual differences in long-term reactions to stressful life experiences among children (16).

The current study examines the longitudinal association of chronic stress exposure indexed by HCC with age at thelarche and TV in a cohort of 997 healthy Chinese boys and girls and extends prior puberty-related gene-byenvironment $(\mathrm{G} \times \mathrm{E})$ work by moving to the polygenic rather than candidate-gene level. We used polygenic risk scores (PRS) derived from a large GWAS meta-analysis of 32 genome-wide association studies in 87,802 women of European descent (14), as well as the first large-scale GWAS of puberty timing in 55,871 males (17).

As to how $\mathrm{G} \times \mathrm{E}$ interaction may manifest, several theoretical models each offer their own prediction. The diathesis stress model predicts that genetic vulnerability, or diathesis, increases the likelihood of a trait in the presence of environmental stress. It also predicts that the heritability of the trait will be higher for children in risk environments $(18,19)$. In contrast, the bioecological model predicts that risk environments will mask genetic differences between children and enriched environments will amplify genetic differences $(20,21)$. The goal of the current article is to provide an empirical test of these two theoretical paradigms that serve as useful heuristics to explain the etiology of early pubertal timing.

\section{Methods}

\section{Study design}

The present study used data of an ongoing longitudinal study examining psychosocial determinants of growth and development in Anhui Province, China. Written informed consent was obtained from parents of all participants. As illustrated in Fig. 1, all the 1263 participants were recruited directly in grade 1 to grade 3 from three large elementary schools of Bengbu city,

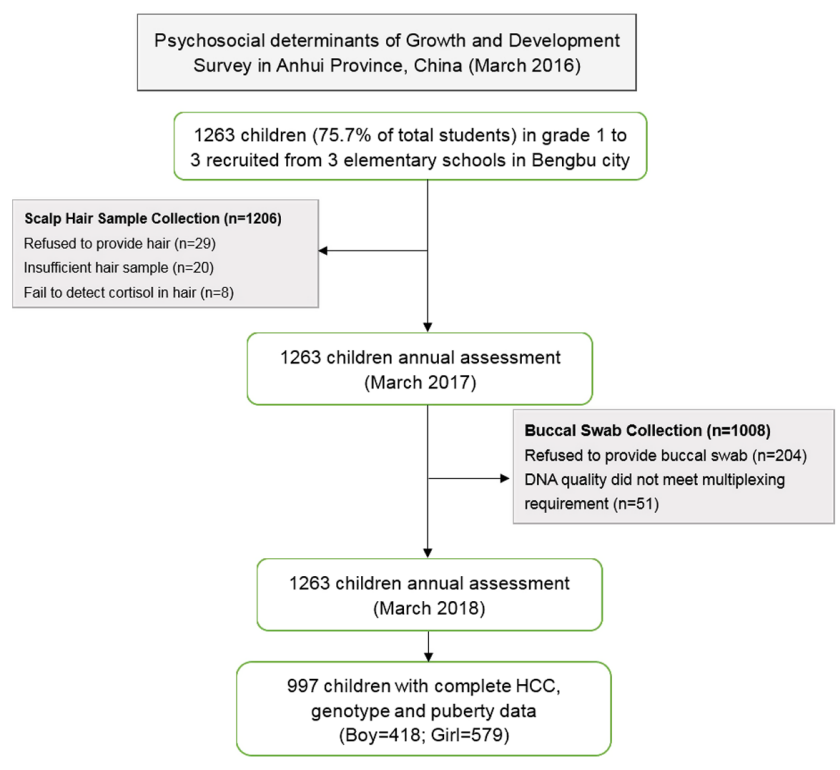

\section{Figure 1}

Flowchart of this study. A full colour version of this figure is available at https://doi.org/10.1530/EJE-19-0033.

Anhui Province, China in March 2016 and were followed annually for physical and pubertal development. The final sample who had available hair cortisol, genotype and pubertal development data consisted of 997 children (418 boys, 579 girls). Of the 57 children who did not have hair cortisol data, 20 did not provide sufficient hair, 29 refused and 19 did not participate for unknown reasons. Three boys were excluded in this analysis due to lack of TV data in all the three waves.

\section{Ethics approval and consent to participate}

Ethical approval was granted by the Institutional Review Boards at Anhui Medical University (No. 20150112), and written informed consent was obtained from parents and school teachers, as well as child assent.

\section{Measurements}

\section{Polygenic risk score (PRS)}

Genotyping and SNP selection DNA extracted from buccal cheek swabs by PCR-RFLP and real-time PCR and genotyped using the Sequeom Mass Array within one batch. The mean concordance rates were $98.8 \%$ for the Sequeom Mass Array Targeted Genotyping System. We chose 11 SNPs for decreasing age at menarche which reached genome-wide significance in a joint analysis of 32 GWAS of 87,802 women of European ancestry (14). One 
SNP (rs5932886) was excluded due to genotyping rate $<10 \%$. We chose another ten single-nucleotide polymorphisms (SNPs) associated with both decreasing age at voice breaking in men and menarche in women from the largest genomic analysis (17). Four out of these 21 SNPs (rs5932886, rs35327298, rs142058842 and rs4237264) were excluded due to MAFs $<5 \%$. LIN28B on chromosome 6 (rs314276) which was the first genetic determinant associated with earlier breast development and voice breaking was selected (22). All the call rate of the 17 SNPs was 99-100\%.

PRS were derived by summing the products of numbers of associated alleles (23). Effect estimates for puberty-SNP associations across the 11 SNPs and heterogeneity between ours and study from Felix et al. are listed in Supplementary Table 1 (see section on supplementary data given at the end of this article).

\section{Pubertal development}

Breast development Breast Tanner stage of each girl was assessed by the same female pediatric endocrinologist. Tanner staging was done by palpation of breast tissue in addition to visual inspection, which follows the same protocol of China Puberty Collaboration Study (24). Onset of breast development was defined as attaining breast stage 2 or greater (B2+).

TV assessment TV was estimated by a trained and qualified male pediatric endocrinologist through palpation to the nearest $1 \mathrm{~mL}$ using Prader's orchidometer (25). In case TVs of the two testes were not equal, the larger testis measurement was used. Onset of testicular development was defined as attaining testicular volume $4 \mathrm{ml}$ or greater (TV4+).

\section{Chronic stress exposure}

Hair cortisol Written instructions were accompanied by several pictures illustrating hair sample collection and preparation. A minimum of $50 \mathrm{mg}$ of hair for a $3 \mathrm{~cm}$ segment from the posterior vertex area of the head was obtained from each participant. Wash and steroid extraction procedures followed the protocol described by Veldhorst et al. (26). A minimum of $10 \mathrm{mg}$ of hair was weighed and put into a glass vial. The samples were dissolved in $250 \mu \mathrm{L}$ phosphate buffer ( $\mathrm{pH} 8$ ) for 1 min to ensure dissolution. The DRG Salivary Cortisol ELISA kit was used to measure cortisol concentrations. The reconstituted sample was measured in duplicate, to assure the reliability of results, using a commercially available luminescence immunoassay with detection (range: 0.005-4 $\mu \mathrm{g} / \mathrm{dL}$ ). Inter-assay coefficient of variation was $7.0 \%$ and intraassay coefficients of variation were $3.6 \%$.

\section{Covariates}

Physical examination Annual physical measurements were made in duplicate during school hours. Height was measured with a portable stadiometer to the nearest $0.1 \mathrm{~cm}$ and weight with an electronic scale (Tanita TI1618) to the nearest $0.1 \mathrm{~kg}$. BMI was calculated as weight (in $\mathrm{kg}$ ) divided by height squared (in $\mathrm{m}$ ). 'The China Body Mass Index Curve for Boys and Girls Aged 0-18 Years' is used to convert BMI values into age-specific BMI percentile levels (27).

Household income Family monthly income was measured with the question from parents: 'How much is the average monthly income for your family?' with six responses alternatives: '<5000 yuan per month (\$ 755.3$)$ ', '5000-10,000 yuan per month (\$755.3-1510.7)', '10,00015000 yuan per month (\$ 1510.7-2266.0)', '15,00020,000 yuan per month (\$2266.0-3021.4)', '20,000-30,000 yuan per month (\$3021.4-4532.1)' and '>30,000 yuan per month (\$4532.1)'.

Parental education level Parents reported their highest level of education using a 5-point scale (1, did not complete high school; 2, high school; 3, college diploma or trades certificate; 4, undergraduate degree and 5, graduate degree).

\section{Analytic strategy}

We explored the breast Tanner stage and TV of adolescents across three repeated assessments in Wave 1 to Wave 3 using hierarchical linear modeling (Stata command: xtmixed, using mixed-effects maximum likelihood regression and unstructured covariance matrix; Stata, version 13 (StataCorp LLC)). The fixed effect of PRS and HCC as both continuous variables and categorically with tertile on the trajectories of breast Tanner stage and TV over three years was determined, adjusting for confounding variables.

For girls who reached breast stage 2 or greater (B2+) at the first study visit (left-censored data), the beginning of the interval was defined as the 3rd percentile value of age from the China Puberty Research Collaboration study data (24), and the end of the interval was the date of the initial research study visit when B2+ was observed. 
For girls who reached B2+ and for boys who reached TV4+ during observed follow-up visits, the interval was defined as the period from the last exam visit consistently at B1 for girls or TV $<4 \mathrm{~mL}$ for boys, to the first visit where the girl or the boy was observed to be consistently at B2+ or TV4+.

Kaplan-Meier analyses were used to determine the effect of PRS and HCC tertile on median age at breast and testicular development, incorporating interval censored data. Weibull accelerated failure time (AFT) models were used to obtain time ratios (TR) with 95\% confidence intervals, which compares the median age at entry into B2+ and TV4+ among girls and boys with the characteristic of interest, or the exposure, to girls and boys in the reference category. This model accounts for varying amounts of time and varying ages of the girls while under observation. A TR of $>1.0$ indicates a longer time to pubertal onset, while a TR of $<1.0$ generally indicates earlier age at pubertal development compared to the reference group (e.g., 9.08 years/9.25 years $=0.982$, representing a 2.0-month earlier). All data were analyzed using Stata version 13. As the sample size of this cohort is slightly small (girls: $n=579$, boys: $n=418$ ). The estimated power and alpha for boys in our study was 0.74 and 0.035 , while for girls it was 0.83 and 0.04 .

\section{Results}

\section{General information}

The cohort included 418 boys and 579 girls aged $8.0 \pm 0.8$ years (6.4-9.8 years old) at baseline. The percentage of boys and girls whose BMI $\geq 95$ th percentile was 22.2 and $21.4 \%$, respectively. Eight boys and two girls had BMI $<5$ th percentile and were included into the $<50$ th percentile group.

Median age for thelarche was 9.22 years. Median age for TV4+ was 11.15 years. Girls with divorced parents matured slightly earlier (1.2 months earlier, time ratio $=0.989, P=0.040)$, while boys with divorced parents matured slightly delayed (1.3 months delayed, time ratio $=1.01, P=0.035)$.

\section{PRS, HCC with breast Tanner stage and TV}

No significant correlation was found between PRS and HCC $(r=0.078, P=0.06)$. Results showed each additional puberty-accelerating allele was associated with 0.02-point increase in breast Tanner stage and $0.05 \mathrm{~mL}$ increase in TV, respectively (Table 1). For each 1-unit increase in HCC, breast Tanner stage and TV increased by 0.03 point and $0.03 \mathrm{~mL}$, respectively.

\section{Association of PRS, HCC tertile with age at thelarche among girls}

The proportion of girls who had attained thelarche during the study varied by PRS and HCC tertile (Table 2). As illustrated in Table 2, girls with high PRS were progressively 1.2 months earlier reaching breast stage 2 than those with low PRS (adjusted TR: 0.989, $P<0.001$ ). Girls experiencing chronic stress (upper tertile of HCC) were more likely to have earlier thelarche compared with moderate amount of stress (median tertile of HCC) (1.0 months earlier, adjusted TR: 0.991, $P=0.013)$.

\section{Association of PRS, HCC tertile with age at testicular volume $\geq 4 \mathrm{~mL}$ (TV4+) among boys}

As illustrated in Table 2, boys with high PRS were progressively more likely to have reached TV4+ than those with low PRS (1.9 months earlier, adjusted TR: 0.986, $P<0001)$. Boys under chronic stress were progressively more likely to have reached TV4+ than those with moderate amount of stress (1.2 months earlier, adjusted TR: 0.991, $P=0.004)$.

\section{Longitudinal effects of chronic stress on age at thelarche and testicular maturation by polygenic risk}

The effects of chronic stress on thelarche were only observed among moderate and low PRS girls, with 1.7 months and 2.2 months earlier, respectively (moderate PRS: adjusted TR: 0.985; $P=0.007$; low PRS: adjusted TR: 0.982; $P<0.001)$. No similar effect was observed among girls with high PRS (adjusted TR: 1.01; $P=0.242$ ) (Fig. 2).

For boys, chronic stress associated with 2.0 months earlier at TV4+ among those with high PRS (adjusted TR: 0.983; $P=0.005$ ) (Fig. 2). For moderate and low PRS boys, similar effects were not observed (both adjusted TR: 0.989; $P>0.05)$.

\section{Longitudinal effects of polygenic influence on age at thelarche and TV4+ under different stress level}

Age at thelarche and TV4+ was compared within different PRS groups under chronic stress and under low level of stress indexed by HCC, as illustrated in Fig. 3. 
Table 1 Effects of polygenic risk score and hair cortisol concentration on breast Tanner stage and testicular volume using hierarchical linear modeling.

\begin{tabular}{|c|c|c|c|c|c|c|c|c|}
\hline \multirow[b]{3}{*}{ Variables } & \multicolumn{4}{|c|}{ Breast Tanner stage } & \multicolumn{4}{|c|}{ Testicular volume } \\
\hline & \multirow[b]{2}{*}{ Adjusted coefficient } & \multicolumn{2}{|c|}{$95 \% \mathrm{Cl}$} & \multirow[b]{2}{*}{$P$ value } & \multirow[b]{2}{*}{ Adjusted coefficient } & \multicolumn{2}{|c|}{$95 \% \mathrm{Cl}$} & \multirow[b]{2}{*}{$P$ value } \\
\hline & & Lower & Upper & & & Lower & Upper & \\
\hline PRS & 0.02 & 0.01 & 0.03 & 0.018 & 0.05 & 0.03 & 0.07 & 0.010 \\
\hline $\mathrm{HCC}$ & 0.03 & 0.01 & 0.04 & 0.001 & 0.03 & 0.01 & 0.06 & 0.008 \\
\hline Age & 0.42 & 0.39 & 0.44 & $<0.001$ & 0.51 & 0.48 & 0.54 & $<0.001$ \\
\hline $\mathrm{BMI}$ & 0.03 & 0.02 & 0.04 & $<0.001$ & -0.01 & -0.04 & -0.01 & 0.364 \\
\hline $\mathrm{PRS} \times \mathrm{HCC}$ & 0.002 & -0.004 & 0.009 & 0.475 & 0.008 & -0.002 & 0.019 & 0.118 \\
\hline
\end{tabular}

Adjusted coefficients are regression coefficient adjusted for household income, parental education and parental divorce.

$\mathrm{BMI}$, body mass index; HCC, hair cortisol concentration; PRS, polygenic risk score.

Results demonstrated evidence for bioecological $\mathrm{G} \times \mathrm{E}$ interaction model among girls, no significant group differences were observed for age at thelarche across PRS groups under chronic stress $(9.10,9.11$ and 9.15 y for high, moderate and low PRS group; $F=2.07, P=0.127$ ), while significant group differences were observed for age at thelarche across PRS groups under low level of stress (9.06, 9.36 and 9.53 years for high, moderate and low PRS, respectively; $F=105.06, P<0.0001$ ).

In contrast to girls, there was evidence for diathesis stress $G \times E$ interaction in boys. Significant differences were observed for age at TV4+ across PRS groups under chronic stress (age at TV4+ was 10.91, 11.06 and 11.17 years for high, moderate and low PRS, respectively; $F=100.48, P<0.0001$ ), while none of the group differences were statistically significant under low level of stress $(F=1.07, P=0.344)$.

\section{Discussion}

Our previous analysis revealed dose-response-like relations between chronic stress indexed by HCC, with advanced pubertal development among boys and girls (7). In the current study, we applied a 3-year longitudinal design, while at the same time introducing gene-environment interaction paradigm, to better understand the effects of chronic stress in the regulation of pubertal timing. By taking into account time elapsed between the chronic stress exposure and the pubertal onset, we provide further evidence of puberty-advancing effects of chronic stress using Weibull AFT model. The present study further tested possible role of genetic variations discovered in GWAS in the susceptibility to early pubertal onset following chronic stress exposure. The findings suggest that chronic

Table 2 Age at thelarche for girls and testicular volume (TV) $\geq 4 \mathrm{~mL}$ for boys by polygenic risk score (PRS) and hair cortisol concentration (HCC) tertile from accelerated failure time model. ${ }^{*}$

\begin{tabular}{|c|c|c|c|c|}
\hline Strata & $\boldsymbol{n}(\%)$ & Median age & Time ratio $(95 \% \mathrm{Cl})$ & $P$ value \\
\hline All girls & 579 & 9.22 & & \\
\hline \multicolumn{5}{|l|}{ PRS tertile } \\
\hline Highest & $192(33.2)$ & 9.12 & $0.989(0.983-0.995)$ & $<0.001$ \\
\hline Median & $190(32.8)$ & 9.23 & $0.997(0.991-1.004)$ & 0.532 \\
\hline Lowest & $197(34.0)$ & 9.30 & 1.00 & \\
\hline \multicolumn{5}{|l|}{ HCC environment } \\
\hline Highest tertile (adverse) & $206(35.6)$ & 9.12 & $0.991(0.986-0.998)$ & 0.013 \\
\hline Lowest tertile (favorable) & $192(33.2)$ & 9.30 & 1.001 (0.995-1.008) & 0.543 \\
\hline Median tertile & $181(31.3)$ & 9.25 & 1.00 & \\
\hline All boys & 418 & 11.15 & & \\
\hline \multicolumn{5}{|l|}{ PRS Tertile } \\
\hline Highest & $130(31.1)$ & 11.06 & $0.986(0.979-0.993)$ & $<0.001$ \\
\hline Median & $143(34.2)$ & 11.18 & $0.998(0.992-1.005)$ & 0.637 \\
\hline Lowest & 145 (34.7) & 11.21 & 1.00 & \\
\hline \multicolumn{5}{|l|}{ HCC environment } \\
\hline Highest tertile (adverse) & $146(32.5)$ & 11.06 & $0.991(0.984-0.997)$ & 0.004 \\
\hline Lowest tertile (favorable) & $133(33.0)$ & 11.24 & $1.004(0.998-1.011)$ & 0.146 \\
\hline Median tertile & $139(34.4)$ & 11.17 & 1.00 & \\
\hline
\end{tabular}



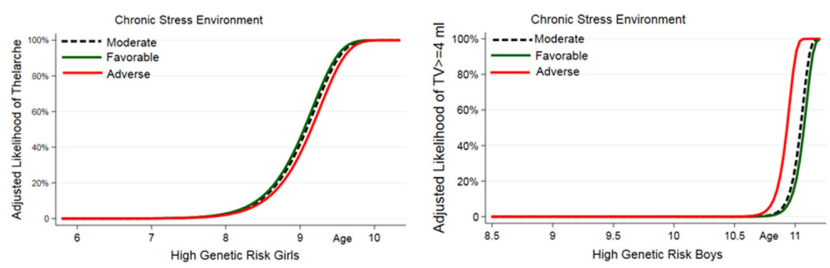

Chronic Stress Environment
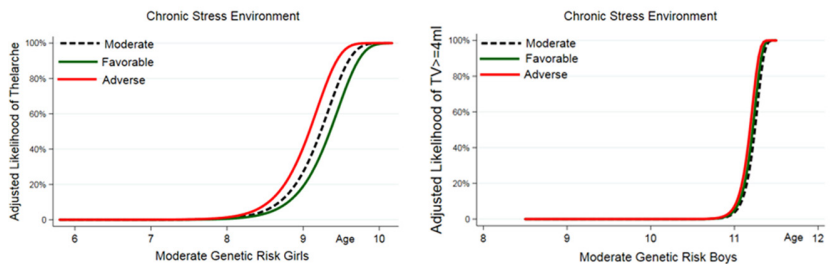

Chronic Stress Environment
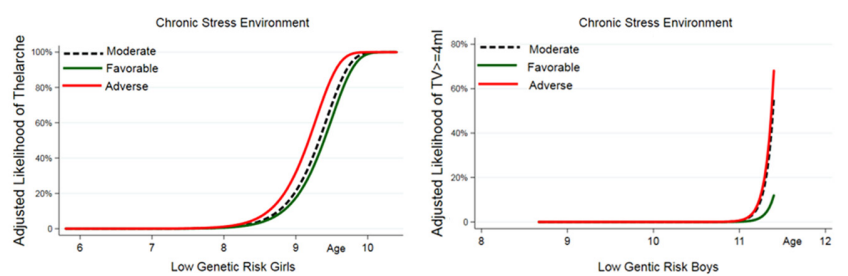

\section{Figure 2}

Comparing the adjusted likelihood of thelarche (left) and testicular volume $\geq 4 \mathrm{~mL}$ (right) among different chronic stress environment for high, moderate and low genetic risk girls and boys. A full colour version of this figure is available at https:// doi.org/10.1530/EJE-19-0033.

stress predict earlier age at pubertal onset in a sex-specific and genetic background-dependent manner. Specifically, for children under chronic stress, early pubertal timing was only observed among girls with moderate (1.7month earlier) and low (2.2-month earlier) polygenic susceptibility, and among boys with high PRS (2.0-month earlier). These results might provide the explanation of why not all chronic stress-exposed children show pubertyadvancing effects highlighted from animal and human studies $(8,9,11,28,29,30,31)$.

The associations between chronic stress with 2-month earlier onset of puberty observed in high PRS boys lends support for diathesis stress or cumulative-risk model (18, 19 ), which suggests that boys carrying additional pubertyadvancing alleles are found to be more likely to mature early when exposed to adverse environment (high chronic stress exposure). To date, studies regarding association between psychosocial stress with pubertal timing among boys is limited. The present study provides an integrated view of $\mathrm{G} \times \mathrm{E}$ interactions in pubertal timing among boys, suggesting that chronic stress confer an extra risk for earlier onset of testicular maturation only for those with genetic predisposition.
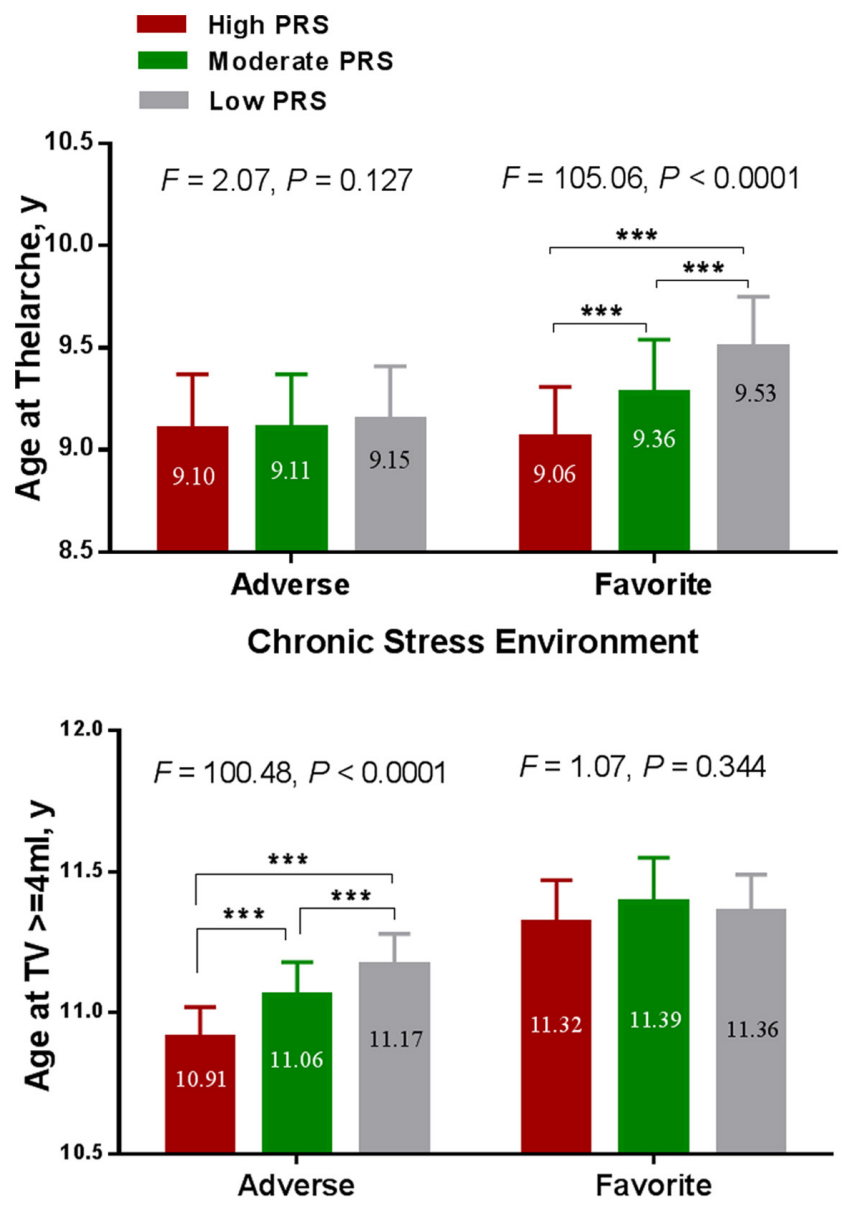

Chronic Stress Environment

\section{Figure 3}

Comparing the age at thelarche (left) and testicular volume (TV) $\geq 4 \mathrm{~mL}$ (right) by PRS tertiles under adverse (highest tertile of HCC) and favorable (lowest tertile of HCC) environment. $\star P<0.05 ; * * P<0.01 ; * \star * P<0.001$. HCC, hair cortisol concentration; PRS, polygenic risk score. A full colour version of this figure is available at https://doi.org/10.1530/EJE-190033.

What we find in girls is more complex and contrasts with that of boys. Puberty-accelerating effects of chronic stress was not observed in high PRS girls, but those with moderate or low PRS (1.7- and 2.2-month earlier, respectively). Further analysis demonstrated that genetic influences on early pubertal timing were most prominent among girls under favorable environment (low chronic stress exposure). These findings echo with the bioecological $G \times E$ interaction model which predicts that adverse environments mask genetic differences between children and enriched environments will enable genetic differences $(20,21)$. 
While needing replication, these findings are important suggesting that the effect of chronic stress on puberty tend to vary depending on the genetic background. One possible explanation is that chronic stress may be more important in pubertal onset for girls who have a low genetic risk than for girls who have a high genetic risk. Fox and Beevers (2016) proposed that certain forms of genetic profile may represent differential biological reactivity to psychosocial stressors (32). It is plausible that polygenic susceptibility for early puberty might involve neurobiological reactivity in the pathway integrating limbic brain stress with hypothalamic reproductive kisspeptin-GnRH systems, in a sexspecific manner. Consistent with this assumption, both physiological variation in autonomic and adrenocortical reactivity to stress (33) and genotypic variation in the estrogen receptor- $\alpha$ gene (ESR1) $(34,35)$ have been found to moderate the effects of psychosocial stress on puberty. Hartman et al. replicated work from Manuck et al. (34) by showing that ESR1 SNPs appear to play a role in determining which girls are more and which are less affected by the developmental experiences with regard to pubertal timing (35).

The explanation for the observed sex difference in the interactive effect of chronic stress and polygenic susceptibility on early pubertal timing is likely complex. To increase survival and reproductive success and perhaps minimize allostatic load, the HPA and the HPG axes work in parallel to fine-tune each other thereby integrating environmental, psychological, reproductive and genetic factors. The HPG and HPA axes both exhibit striking sex differences, which are organized and activated by dynamic changes in stress hormones across the lifespan. Thus, possible sex differences in moderation effects of adversity on HPA-HPG coupling may be considered. For example, Simmons et al. reported HPA-HPG coupling was positive only in girls from low-to-moderate adversity backgrounds and that HPA-HPG functioning was uncoupled in girls from high-adversity backgrounds (36). While sex hormones (testosterone and estrogen) are the primary drivers of differential gene expression, these hormones do not explain all sex differences, pointing toward an alternative mechanism prior gonadal steroid synthesis. New advances in understanding how environmentally sensitive, yet highly heritable pubertal timing is regulated have come from the field of epigenetics $(37,38,39)$. Epigenetic mechanisms are likely to play a large role in how the gene-environment interactions operate differently in regulating pubertal timing for boys and girls (40). Recent studies suggest the involvement of an epigenetically mediated control of neuroendocrinerelated gene transcription in reactivation of the HPG axis (41). In addition, accumulating evidence suggests that sex-specific changes in methylation patterns across the window of puberty and that girls are particularly sensitive to the effects of DNA methylation changes (42), sex-typical pattern of epigenetic modifications across the genome, such as sex-specific DNA methylation in response to environmental cues, might in part contribute to the sex differences observed in our study. Future research probing these areas will provide new and exciting mechanisms and avenues for exploration of sex differences in the functional cross-talk between chronic stress with sexual maturation.

\section{Limitations}

Several limitations of the current study warrant consideration. First, although the genetic architecture underlying pubertal timing is shared between the sexes, as all the selected alleles in this study have concordant accelerating effect in males and females, they might have different effect sizes. Besides, PRS derived from a discovery GWAS of Europeans might not be informative predictors in Asian population. Thirdly, the present report only evaluates HCC at baseline, limiting the ability to track psychosocial burden over time. It should furthermore be considered that interpretation of hair cortisol levels is complex, because cumulative cortisol secretions are a function of multiple, potentially interacting factors, including hair washing frequency, medication use, genetic dispositions, developmental experiences and different analysis methodologies (43). It should be noted that our dataset was fairly small, and the majority of the boys were in the early stages of puberty, which may have limited power to detect $\mathrm{G} \times \mathrm{E}$ effects. Also, it would be reasonable to perform pubertal assessments every 3-6 months rather than once a year. Pubertal onset lies within an interval instead of being observed exactly, which often leads to interval censoring. Finally, our preliminary investigation did not use the GWAS-driven pathway analysis, thus suffered difficulty in elucidating possible molecular pathways for the accelerating effects of chronic stress. With the discovery of genome-wide significant common variants, we look forward to studies that discover the functional variants responsible for these findings. 


\section{Conclusion}

The present study demonstrates a required role of genetic background in mediating chronic stress induced early pubertal timing. These data enhance our understanding of the cross-talk between the genome and the environment in coregulating pubertal timing, permitting a better interpretation of marked variability in response among children exposed to the same environmental risk. More in-depth studies of gene-by-environment interactions in the light of epigenetics are necessary to understand phenotypic plasticity in pubertal timing.

\section{Supplementary data}

This is linked to the online version of the paper at https://doi.org/10.1530/ EJE-19-0033.

\section{Declaration of interest}

The authors declare that there is no conflict of interest that could be perceived as prejudicing the impartiality of this study.

\section{Funding}

This work was supported by the National Natural Science Foundation of China (grant numbers 81872638) and Natural Science Foundation of Anhui Province for Distinguished Young Scholars (grant numbers 1908085J26).

\section{Author contribution statement}

Conceptualization and design: Ying Sun, Fangbiao Tao. Acquisition of data: Jiao Fang, Yuhui Wan, Puyu Su. Analysis and interpretation of data: Ying Sun, Jiao Fang, Yuhui Wan, Puyu Su. Revising it for intellectual content: Ying Sun, Fangbiao Tao, Puyu Su, Yuhui Wan. Final approval of completed article: All authors.

\section{Acknowledgments}

The authors are grateful to the local principal investigators and staff, as well as all the study participants, their teachers and their parents.

\section{References}

1 Golub MS, Collman GW, Foster PM, Kimmel CA, Rajpert-De Meyts E, Reiter EO, Sharpe RM, Skakkebaek NE \& Toppari J. Public health implications of altered puberty timing. Pediatrics 2008121 (Supplement 3) S218-S230. (https://doi.org/10.1542/peds.20071813G)

2 Day FR, Bulik-Sullivan B, Hinds DA, Finucane HK, Murabito JM, Tung JY, Ong KK \& Perry JRB. Shared genetic aetiology of puberty timing between sexes and with health-related outcomes. Nature Communications 20156 8842. (https://doi.org/10.1038/ncomms9842)

3 Avendaño MS, Vazquez MJ \& Tena-Sempere M. Disentangling puberty: novel neuroendocrine pathways and mechanisms for the control of mammalian puberty. Human Reproduction Update 201723 737-763. (https://doi.org/10.1093/humupd/dmx025)

4 Parent AS, Franssen D, Fudvoye J, Pinson A \& Bourguignon JP. Current changes in pubertal timing: revised vision in relation with environmental factors including endocrine disruptors. Endocrine Development 201629 174-184. (https://doi.org/10.1159/000438885)

5 Villamor E \& Jansen EC. Nutritional determinants of the timing of puberty. Annual Review of Public Health 201637 33-46. (https://doi. org/10.1146/annurev-publhealth-031914-122606)

6 Hiatt RA, Stewart SL, Hoeft KS, Kushi LH, Windham GC, Biro FM, Pinney SM, Wolff MS, Teitelbaum SL \& Braithwaite D. Childhood socioeconomic position and pubertal onset in a cohort of multiethnic girls: implications for breast cancer. Cancer Epidemiology, Biomarkers and Prevention 201726 1714-1721. (https://doi org/10.1158/1055-9965.EPI-17-0496)

7 Sun Y, Xu YY, Hu JJ \& Tao FB. Association of a chronic stress biomarker with advanced development of breast and testicular volume. JAMA Pediatrics 2017171 596-598. (https://doi.org/10.1001/ jamapediatrics.2017.0038)

8 Sun Y, Mensah FK, Azzopardi P, Patton GC \& Wake M. Childhood social disadvantage and pubertal timing: a national birth cohort from Australia. Pediatrics 2017139 e20164099. (https://doi. org/10.1542/peds.2016-4099)

9 Kelly Y, Zilanawala A, Sacker A, Hiatt R \& Viner R. Early puberty in 11-year-old girls: millennium cohort study findings. Archives of Disease in Childhood 2017102 232-237. (https://doi.org/10.1136/ archdischild-2016-310475)

10 Hochberg Z \& Belsky J. Evo-devo of human adolescence: beyond disease models of early puberty. BMC Medicine 201311 113. (https:// doi.org/10.1186/1741-7015-11-113)

11 Li L, Denholm R \& Power C. Child maltreatment and household dysfunction: associations with pubertal development in a British birth cohort. International Journal of Epidemiology 201443 1163-1173. (https://doi.org/10.1093/ije/dyu071)

12 Day FR, Thompson DJ, Helgason H, Chasman DI, Finucane H, Sulem P, Ruth KS, Whalen S, Sarkar AK, Albrecht E et al. Genomic analyses identify hundreds of variants associated with age at menarche and support a role for puberty timing in cancer risk. Nature Genetics 201749 834-841. (https://doi.org/10.1038/ng.3841)

13 Perry JR, Day F, Elks CE, Sulem P, Thompson DJ, Ferreira T, He C, Chasman DI, Esko T, Thorleifsson G et al. Parent-of-origin-specific allelic associations among 106 genomic loci for age at menarche. Nature 2014514 92-97. (https://doi.org/10.1038/nature13545)

14 Elks CE, Perry JR, Sulem P, Chasman DI, Franceschini N, He C, Lunetta KL, Visser JA, Byrne EM, Cousminer DL et al. Thirty new loci for age at menarche identified by a meta-analysis of genome-wide association studies. Nature Genetics 201042 1077-1085. (https://doi. org/10.1038/ng.714)

15 Stalder T, Steudte-Schmiedgen S, Alexander N, Klucken T, Vater A, Wichmann S, Kirschbaum C \& Miller R. Stress-related and basic determinants of hair cortisol in humans: a metaanalysis. Psychoneuroendocrinology 201777 261-274. (https://doi. org/10.1016/j.psyneuen.2016.12.017)

16 Gray NA, Dhana A, Van Der Vyver L,Van Wyk J, Khumalo NP \& Stein DJ. Determinants of hair cortisol concentration in children: a systematic review. Psychoneuroendocrinology $2018 \mathbf{8 7} 204-214$. (https://doi.org/10.1016/j.psyneuen.2017.10.022)

17 Day FR, Bulik-Sullivan B, Hinds DA, Finucane HK, Murabito JM, Tung JY, Ong KK \& Perry JRB. Shared genetic aetiology of puberty timing between sexes and with health-related outcomes. Nature Communications 20156 8842. (https://doi.org/10.1038/ncomms9842)

18 Monroe SM \& Simons AD. Diathesis-stress theories in the context of life stress research: implications for the depressive disorders. Psychological Bulletin 1991110 406-425. (https://doi. org/10.1037/0033-2909.110.3.406)

19 Zuckerman M. Vulnerability to Psychopathology: A Biosocial Model. Washington, DC: American Psychological Association, 1999.

20 Bronfenbrenner U \& Ceci SJ. Nature-nurture reconceptualized in developmental perspective: a bioecological model. Psychological 
Review 1994101 568-586. (https://doi.org/10.1037/0033295X.101.4.568)

21 Pennington BF, McGrath LM, Rosenberg J, Barnard H, Smith SD, Willcutt EG, Friend A, Defries JC \& Olson RK. Gene x environment interactions in reading disability and attention-deficit/hyperactivity disorder. Developmental Psychology 200945 77-89. (https://doi. org/10.1037/a0014549)

22 Ong KK, Elks CE, Li S, Zhao JH, Luan J, Andersen LB, Bingham SA, Brage S, Smith GD, Ekelund U et al. Genetic variation in LIN28B is associated with the timing of puberty. Nature Genetics $2009 \mathbf{4 1}$ 729-733. (https://doi.org/10.1038/ng.382)

23 Belsky DW, Moffitt TE \& Caspi A. Genetics in population health science: strategies and opportunities. American Journal of Public Health 2013103 S73-S83. (https://doi.org/10.2105/AJPH.2012.301139)

24 Sun Y, Tao FB, Su PY, Mai JC, Shi HJ, Han YT, Wang H, Lou XM, Han J \& Liu J. National estimates of the pubertal milestones among urban and rural Chinese girls. Journal of Adolescent Health $2012 \mathbf{5 1}$ 279-284. (https://doi.org/10.1016/j.jadohealth.2011.12.019)

25 Zachmann M, Prader A, Kind HP, Häfliger H \& Budliger H. Testicular volume during adolescence. Cross-sectional and longitudinal studies. Helvetica Paediatrica Acta 197429 61-72.

26 Vanaelst B, Huybrechts I, Bammann K, Michels N, de Vriendt T, Vyncke K, Sioen I, Iacoviello L, Günther K, Molnar D et al. Intercorrelations between serum, salivary, and hair cortisol and child-reported estimates of stress in elementary school girls. Psychophysiology 201249 1072-1081. (https://doi.org/10.1111/ j.1469-8986.2012.01396.x)

27 Li H, Zong XN, Ji CY \& Mi J. Body mass index cut-offs for overweight and obesity in Chinese children and adolescents aged 2-18 years. Zhonghua Liu Xing Bing Xue Za Zhi 201031 616-620. (https://doi. org/10.3760/cma.j.issn.0254-6450.2010.06.004)

28 Magnus MC, Anderson EL, Howe LD, Joinson CJ, PentonVoak IS \& Fraser A. Childhood psychosocial adversity and female reproductive timing: a cohort study of the ALSPAC mothers. Journal of Epidemiology and Community Health 201872 34-40. (https://doi. org/10.1136/jech-2017-209488)

29 Ross JW, Hale BJ, Seibert JT, Romoser MR, Adur MK, Keating AF $\&$ Baumgard LH. Physiological mechanisms through which heat stress compromises reproduction in pigs. Molecular Reproduction and Development 201784 934-945. (https://doi.org/10.1002/mrd.22859)

30 Culpin I, Heron J, Araya R, Melotti R, Lewis G \& Joinson C. Father absence and timing of menarche in adolescent girls from a UK cohort: the mediating role of maternal depression and major financial problems. Journal of Adolescence 201437 291-301. (https:// doi.org/10.1016/j.adolescence.2014.02.003)

31 Boynton-Jarrett R, Wright RJ, Putnam FW, Lividoti Hibert E, Michels KB, Forman MR \& Rich-Edwards J. Childhood abuse and age at menarche. Journal of Adolescent Health 201352 241-247. (https:// doi.org/10.1016/j.jadohealth.2012.06.006)

32 Fox E \& Beevers CG. Differential sensitivity to the environment: contribution of cognitive biases and genes to psychological wellbeing. Molecular Psychiatry 201621 1657-1662. (https://doi. org/10.1038/mp.2016.114)

33 Ellis BJ, Shirtcliff EA, Boyce WT, Deardorff J \& Essex MJ. Quality of early family relationships and the timing and tempo of puberty: effects depend on biological sensitivity to context. Development and Psychopathology 201123 85-99. (https://doi.org/10.1017/ S0954579410000660)

34 Hartman S, Widaman KF \& Belsky J. Genetic moderation of effects of maternal sensitivity on girl's age of menarche: replication of the Manuck et al. study. Development and Psychopathology 201527 747-756. (https://doi.org/10.1017/S0954579414000856)

35 Manuck SB, Craig AE, Flory JD, Halder I \& Ferrell RE. Reported early family environment covaries with menarcheal age as a function of polymorphic variation in estrogen receptor- $\alpha$. Development and Psychopathology 201123 69-83. (https://doi.org/10.1017/ S0954579410000659)

36 Simmons JG, Byrne ML, Schwartz OS, Whittle SL, Sheeber L, Kaess M, Youssef GJ \& Allen NB. Dual-axis hormonal covariation in adolescence and the moderating influence of prior trauma and aversive maternal parenting. Developmental Psychobiology 201557 670-687. (https://doi.org/10.1002/dev.21275)

37 Rzeczkowska PA, Hou H, Wilson MD \& Palmert MR. Epigenetics: a new player in the regulation of mammalian puberty. Neuroendocrinology 201499 139-155. (https://doi. org/10.1159/000362559)

38 Lomniczi A \& Ojeda SR. The emerging role of epigenetics in the regulation of female puberty. Endocrine Development 201629 1-16. (https://doi.org/10.1159/000438840)

39 Maruska KP \& Fernald RD. Social regulation of gene expression in the hypothalamic-pituitary-gonadal axis. Physiology 201126 412-423. (https://doi.org/10.1152/physiol.00032.2011)

40 Cousminer DL, Widén E \& Palmert MR. The genetics of pubertal timing in the general population: recent advances and evidence for sex-specificity. Current Opinion in Endocrinology, Diabetes, and Obesity 201623 57-65. (https://doi.org/10.1097/MED.0000000000000213)

41 Almstrup K, Lindhardt Johansen M, Busch AS, Hagen CP, Nielsen JE, Petersen JH \& Juul A. Pubertal development in healthy children is mirrored by DNA methylation patterns in peripheral blood. Scientific Reports 20166 28657. (https://doi.org/10.1038/srep28657)

42 Thompson EE, Nicodemus-Johnson J, Kim KW, Gern JE, Jackson DJ, Lemanske RF \& Ober C. Global DNA methylation changes spanning puberty are near predicted estrogen-responsive genes and enriched for genes involved in endocrine and immune processes. Clinical Epigenetics 201810 62. (https://doi.org/10.1186/s13148-018-0491-2)

43 Wester VL \& van Rossum EF. Clinical applications of cortisol measurements in hair. European Journal of Endocrinology 2015173 M1-M10. (https://doi.org/10.1530/EJE-15-0313)

44 Biro FM, Greenspan LC, Galvez MP, Pinney SM, Teitelbaum S, Windham GC, Deardorff J, Herrick RL, Succop PA, Hiatt RA et al. Onset of breast development in a longitudinal cohort. Pediatrics 2013 132 1019-1027. (https://doi.org/10.1542/peds.2012-3773)

Received 16 January 2019

Revised version received 27 April 2019

Accepted 29 May 2019 